\title{
CC chemokine ligands in patients presenting with stable chest pain: association with atherosclerosis and future cardiovascular events
}

\author{
M. O. Versteylen ${ }^{1}$ M. Manca ${ }^{2}$ I. A. Joosen ${ }^{3}$ D. E. Schmidt ${ }^{2}$ M. Das ${ }^{4}$ L. Hofstra ${ }^{5}$ H. J. Crijns ${ }^{3}$. \\ E. A. Biessen ${ }^{2}$ B. L. Kietselaer ${ }^{6}$
}

Published online: 29 August 2016

(C) The Author(s) 2016. This article is available at SpringerLink with Open Access.

\begin{abstract}
Background CC chemokine ligands (CCLs) are elevated during acute coronary syndrome (ACS) and correlate with secondary events. Their involvement in plaque inflammation led us to investigate whether CCL3-5-18 are linked to the extent of coronary artery disease (CAD) and prognostic for primary events during follow-up.

Methods We measured CCL3-5-18 serum concentrations in 712 patients with chest discomfort referred for cardiac CT angiography. Obstructive CAD was defined as $\geq 50 \%$ stenosis. The extent of CAD was measured by calcium score and segment involvement score (number of coronary segments with any CAD, range 0-16). Patients were followed up for all-cause mortality, ACS and revascularisation, for a mean $26 \pm 7$ months.

Results Patients with obstructive CAD had significantly higher CCL5 $(p=0.02)$, and borderline significantly elevated CCL18 plasma levels as compared with patients with
\end{abstract}

M. O. Versteylen

mathijs.versteylen@gmail.com

1 Cardiovascular Research Institute Maastricht (CARIM)/Department of Cardiology, Maastricht University Medical Center, Maastricht, The Netherlands

2 Department of Pathology, Maastricht University Medical Center, Maastricht, The Netherlands

3 Department of Cardiology, Maastricht University Medical Center, Maastricht, The Netherlands

4 Cardiovascular Research Institute Maastricht (CARIM)/Department of Radiology, Maastricht University Medical Center, Maastricht, The Netherlands

5 Cardiology Center Netherlands, Utrecht, The Netherlands

6 Department of Cardiology and Radiology, Maastricht University Medical Center, Maastricht, The Netherlands
$<50 \%$ stenosis $(p=0.06)$. CCL18 levels were associated with coronary calcification $(p=0.002)$ and segment involvement score $(p=0.007)$. Corrected for traditional risk factors, only CCL5 provided independent predictive value for obstructive CAD: odds ratio (OR) 1.27 (1.02-1.59), $p=$ 0.04 . CCL5 provided independent predictive value for primary events during follow-up: OR 1.62 (1.03-2.57), $p=$ 0.04 .

Conclusions While CCL18 serum levels correlated with extent of CAD, CCL5 demonstrated an independent association with the presence of obstructive CAD, and occurrence of primary cardiac events.

Keywords CC chemokine ligands - Cardiac computed tomographic angiography · Coronary artery disease . Cardiac events · Prognosis

\section{Introduction}

Atherosclerosis is generally regarded as a dyslipidaemic disorder with a strong inflammatory character [1]. This inflammation process is in part directed by chemokines, which play a role in mediating leukocyte recruitment to sites of injury, vascular smooth muscle cell proliferation, neovascularisation and platelet activation [2, 3]. Among others, CC chemokine ligand (CCL) 3, 5 and 18 have been detected in human atherosclerotic lesions [4, 5].

In addition, CCL3, 5 and 18 have been associated with acute coronary syndrome (ACS) and refractory unstable angina pectoris (UAP) in several prospective studies. In patients with UAP, CCL5 and 18 were transiently raised during UAP and indicative of refractory syndromes [6]. CCL3 levels were significantly elevated in patients with acute myocardial infarction (AMI) as compared with con- 
trols, as well as transiently elevated in UAP patients. In addition, CCL3 elevation was associated with future ACS [7]. Also, in a larger cohort of patients presenting with ACS, CCL3, 5 and 18 were independently associated with fatal events during follow-up [8]. Moreover, macrophage expression of CCL18 in patients with atherosclerosis was significantly higher as compared with controls, suggesting CCL18 has a role in atherosclerotic plaque formation [9]. Nevertheless, several other studies investigating different populations reported no association between CCL5 and future cardiovascular events [10-12].

Although CCL3, 5 and 18 might contribute to plaque formation, and could therefore represent attractive therapeutic targets, evidence is conflicting and their association with presence and extent of coronary artery disease (CAD) is not well understood. We hypothesised that CCL3, 5 and 18 are associated with both CAD and cardiac events in patients with stable chest pain.

The goal of this study was therefore to address the relationship between CCL3, 5 and 18 and the presence and extent of CAD as defined by coronary computed tomography angiography (CCTA) in a cohort of patients with stable chest pain. In addition, the association of CCL3, 5 and 18 with primary cardiac events during follow-up was investigated.

\section{Methods}

\section{Study population}

We analysed a cohort of patients who were referred from the outpatient clinic for CCTA to rule out CAD between January 2008 and June 2010. Included were patients who underwent both a calcium score scan and CT angiography. In total 1096 patients were scanned. Excluded were 117 patients who had an inconclusive CCTA, and 95 patients because of missing clinical data. Additionally, 172 blood samples were haemolytic and were excluded from this anal- ysis. Eventually, 712 patients participated in this study and plasma was screened for CCL3, 5 and 18 levels. All patients signed informed consent. The study protocol conforms to the ethical guidelines of the 1975 Declaration of Helsinki, as reflected in approval by the Institutional Review Board and Ethics Committee (MEC 08-4-057).

\section{CCTA acquisition}

CCTA was performed using a 64-slice multidetector row computed tomography scanner (Brilliance 64; Philips Healthcare, Best, the Netherlands) with a $64 \times 0.625 \mathrm{~mm}$ slice collimation and a rotation time of $420 \mathrm{~ms}$. In addition, a native scan using $120 \mathrm{kV}$ and $3 \mathrm{~mm}$ slice thickness was performed to determine the coronary calcium score (Agatston score). The mean total estimated radiation dose was calculated by multiplying the dose-length product by the conversion factor of $0.014 \mathrm{mSv} / \mathrm{mGy} / \mathrm{cm}$ for the thorax [13].

\section{CCTA assessment}

The Agatston method was used to quantify the coronary calcium score [14]. CT angiograms were independently analysed by a cardiologist and a radiologist, both experienced in reading cardiac CT and blinded to clinical information, using the American Heart Association 16 coronary segments model [15]. In case of disagreement, consensus was reached by discussion between the two readers. The degree of stenosis was defined visually and a lesion severity score was calculated as follows: no lesion (score 0), mild diameter stenosis $(<50 \%$, score 1$)$, moderate $(50-70 \%$, score 2$)$ and severe diameter stenosis $(>70 \%$, score 3$)$. This resulted in a lesion severity score ranging from $0-3$ for each individual patient. The presence of one or more lesions with $\geq 50 \%$ stenosis was regarded as significant CAD. In addition, the segment involvement score was defined as the total number of coronary segments with detectable CAD (range 0-16) [16].
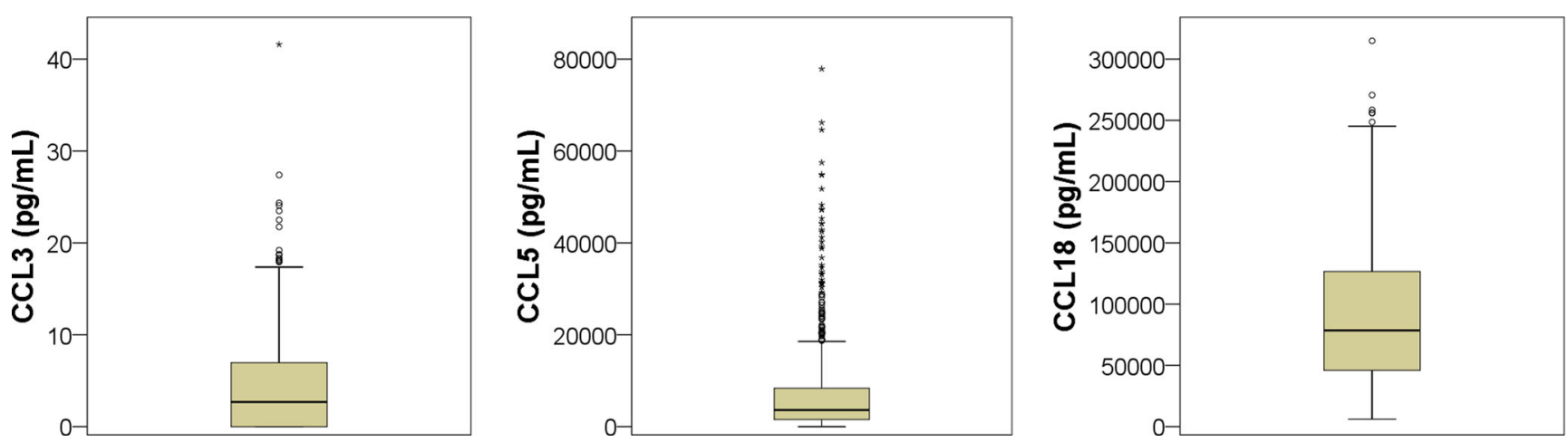

Fig. 1 Median concentrations of CCL3, CCL5 and CCL18 


\section{Biomarker assessment}

Blood samples were collected by ethylenediaminetetraacetic acid (EDTA) coated Vacutainer venipuncture just before the scan. Samples were kept at $4{ }^{\circ} \mathrm{C}$ and centrifuged at $4100 \mathrm{rpm}$ for 10 minutes, and then stored at $-80{ }^{\circ} \mathrm{C}$ until analysis, all within two hours. High-density lipoprotein cholesterol was measured using Synchron LX20 (Beckman Coulter). Low-density lipoprotein cholesterol was calculated using the Friedewald equation, or Cobas Mira Plus (Roche Diagnostics). High-sensitivity C-reactive protein (hsCRP) was measured on BN ProSpec using the CardioPhase hsCRP assay (Siemens Diagnostics).

\section{CCL assessment}

Baseline blood specimens were collected from storage in July 2012. The sera were snap frozen and stored at $-70{ }^{\circ} \mathrm{C}$ until further analysis and underwent only one freeze-thawing cycle. Baseline serum levels of CCL3/MIP-1 $\alpha$, CCL5/ RANTES and CCL18/PARC were determined by commercially available ELISA kits from R\&D systems (Abingdon, United Kingdom; Human CCL3/MIP-1 alpha Quantikine ELISA Kit, $\leq 8.9 \%$ intra-assay variation, Human CCL 5/RANTES DuoSet and Human CCL18/PARC DuoSet). CCL3 ELISA was performed according to the manufacturer's instructions. For CCL5 measurements, $0.75 \mu \mathrm{g} / \mathrm{ml}$ capture antibody and $10 \mathrm{ng} / \mathrm{ml}$ detection antibody were used, for CCL18 we used $1.5 \mu \mathrm{g} / \mathrm{ml}$ capture antibody and $200 \mathrm{ng} / \mathrm{ml}$ detection antibody.

\section{Follow-up}

Patients were followed up for the occurrence of all-cause mortality, ACS (including myocardial infarction) and revascularisation procedures based on conventional coronary angiography including percutaneous coronary intervention (PCI) and coronary artery bypass grafting (CABG), for a mean of $26 \pm 7$ months. Electronic patient records were checked, ACS was defined as typical angina pectoris and troponin T elevation ( $>0.01 \mu \mathrm{g} / \mathrm{l})$ and ST-segment elevation/ depression of $\geq 1 \mathrm{~mm}$, or at least two of these symptoms together with invasive angiographic confirmation of a culprit lesion [17]. Further, we censored follow-up after the first endpoint so that the recorded ACS was not a complication of revascularisation therapy.

\section{Statistics}

Data were analysed using SPSS 22.0 (SPSS Inc., Chicago, IL, USA). Continuous variables were presented as mean \pm standard deviation or median and interquartile range, whether they were of normal or nonparametric distribution, respectively. Differences were assessed using Student's t-test for normally distributed data, or Kruskal-Wallis test for nonparametric distributions. Proportions (\%) were used for categorical values, in which differences were assessed using Fisher's exact test. In regression models, base-10 logarithmic transformation was used in order to include continuous variables with a non-normal distribution. All

Table 1 Baseline characteristics

\begin{tabular}{|c|c|c|c|}
\hline & $\begin{array}{l}<50 \% \text { stenosis } \\
n=509\end{array}$ & $\begin{array}{l}\geq 50 \% \text { stenosis } \\
n=203\end{array}$ & $\begin{array}{l}p \text {-value } \\
-\end{array}$ \\
\hline$\overline{\text { Age (years) }}$ & $54.7 \pm 10.2$ & $60.9 \pm 9.3$ & $<0.001$ \\
\hline Male gender & $266(52.3 \%)$ & $132(65.0 \%)$ & 0.002 \\
\hline Smoking & $107(21.0 \%)$ & $62(30.5 \%)$ & 0.008 \\
\hline Diabetes mellitus & $41(8.1 \%)$ & $17(8.4 \%)$ & 0.88 \\
\hline Family history & $187(36.7 \%)$ & $85(41.9 \%)$ & 0.23 \\
\hline Systolic blood pressure $(\mathrm{mm} \mathrm{Hg})$ & $140 \pm 17$ & $147 \pm 20$ & $<0.001$ \\
\hline BMI $\left(\mathrm{kg} / \mathrm{m}^{2}\right)$ & $26.9 \pm 4.1$ & $27.4 \pm 4.4$ & 0.14 \\
\hline LDL cholesterol (mmol/l) & $3.3 \pm 1.0$ & $3.3 \pm 1.2$ & 0.67 \\
\hline HDL cholesterol (mmol/l) & $1.3 \pm 0.5$ & $1.3 \pm 0.8$ & 0.51 \\
\hline Statin use & $167(33.0 \%)$ & $100(49.5 \%)$ & $<0.001$ \\
\hline Typical chest pain & $59(11.6 \%)$ & $29(14.3 \%)$ & 0.32 \\
\hline Calcium score & $0(0-24.5)$ & $201(53.5-455)$ & $<0.001$ \\
\hline Involvement score & $0(0-2)$ & $5(3-7)$ & $<0.001$ \\
\hline CCL3 (pg/ml) & $2.5(0.0-7.0)$ & $2.8(0.4-7.0)$ & 0.37 \\
\hline CCL5 $\left(\mathrm{pg} / \mathrm{ml} * 10^{2}\right)$ & $33.4(14.5-77.0)$ & $40.7(18.5-109.1)$ & 0.02 \\
\hline $\operatorname{CCL} 18\left(\mathrm{pg} / \mathrm{ml} * 10^{2}\right)$ & $747.1(450.5-1241.3)$ & $885.1(502.5-1331.8)$ & 0.06 \\
\hline hsCRP (mg/dl) & $1.4(0.7-1.4)$ & $1.7(0.9-4.1)$ & 0.02 \\
\hline
\end{tabular}

Values are presented as numbers (\%), means \pm standard deviation or medians (IQR). Significant $p$-values are in bold. $B M I$ body mass index, $C C L$ $C C$ chemokine ligand, $h s C R P$ high-sensitive C-reactive protein 
Table 2 Univariable regression analysis to predict for extent of CAD

\begin{tabular}{|c|c|c|c|}
\hline \multicolumn{4}{|c|}{ Coronary calcium score (log-transformed) } \\
\hline & B-value & $95 \% \mathrm{CI}$ & $p$-value \\
\hline$\overline{\text { Age (years) }}$ & 0.44 & $0.04-0.05$ & $<0.001$ \\
\hline Male gender & 0.14 & $0.15-0.47$ & $<0.001$ \\
\hline Smoking & 0.05 & $-0.05-0.33$ & 0.15 \\
\hline Diabetes mellitus & 0.10 & $0.12-0.70$ & 0.006 \\
\hline Family history & 0.01 & $-0.14-0.20$ & 0.72 \\
\hline Systolic blood pressure (mm Hg) & 0.23 & $0.01-0.02$ & $<0.001$ \\
\hline $\operatorname{BMI}\left(\mathrm{kg} / \mathrm{m}^{2}\right)$ & 0.06 & $-0.00-0.04$ & 0.09 \\
\hline LDL cholesterol (mmol/l) & -0.05 & $-0.13-0.02$ & 0.17 \\
\hline HDL cholesterol (mmol/l) & -0.01 & $-0.16-0.12$ & 0.75 \\
\hline Statin use & 0.19 & $0.27-0.59$ & $<0.001$ \\
\hline Typical chest pain & -0.01 & $-0.28-0.20$ & 0.75 \\
\hline Lg10 CCL3 & 0.04 & $-0.09-0.28$ & 0.33 \\
\hline Lg10 CCL5 & -0.02 & $-0.11-0.07$ & 0.60 \\
\hline Lg10 CCL18 & 0.12 & $0.16-0.73$ & 0.002 \\
\hline Lg10 hsCRP & 0.03 & $-0.12-0.25$ & 0.50 \\
\hline$\geq 50 \%$ stenosis & 0.57 & $1.23-1.52$ & $<0.001$ \\
\hline \multicolumn{4}{|l|}{ Involvement score (log-transformed) } \\
\hline & B-value & $95 \% \mathrm{CI}$ & $p$-value \\
\hline Age (years) & 0.38 & $0.01-0.02$ & $<0.001$ \\
\hline Male gender & 0.20 & $0.09-0.19$ & $<0.001$ \\
\hline Smoking & 0.09 & $0.01-0.13$ & 0.02 \\
\hline Diabetes mellitus & 0.10 & $0.03-0.22$ & 0.01 \\
\hline Family history & 0.03 & $-0.03-0.07$ & 0.44 \\
\hline Systolic blood pressure (mm Hg) & 0.23 & $0.00-0.01$ & $<0.001$ \\
\hline $\operatorname{BMI}\left(\mathrm{kg} / \mathrm{m}^{2}\right)$ & 0.03 & $-0.00-0.01$ & 0.39 \\
\hline LDL cholesterol (mmol/l) & 0.00 & $-0.02-0.03$ & 0.94 \\
\hline HDL cholesterol (mmol/l) & -0.01 & $-0.05-0.04$ & 0.80 \\
\hline Statin use & 0.19 & $0.09-0.19$ & $<0.001$ \\
\hline Typical chest pain & -0.00 & $-0.08-0.08$ & 0.97 \\
\hline Lg10 CCL3 & 0.04 & $-0.03-0.10$ & 0.25 \\
\hline Lg10 CCL5 & -0.03 & $-0.04-0.02$ & 0.46 \\
\hline Lg10 CCL18 & 0.10 & $0.03-0.22$ & 0.007 \\
\hline Lg10 hsCRP & 0.03 & $-0.04-0.08$ & 0.56 \\
\hline$\geq 50 \%$ stenosis & 0.66 & $0.47-0.55$ & $<0.001$ \\
\hline
\end{tabular}

Univariable linear regression model. $B M I$ body mass index, $C C L$ CC chemokine ligand, $h s C R P$ high-sensitive C-reactive protein

Table 3 Multivariable logistic regression analysis to predict for CAD $(\geq 50 \%$ stenosis)

\begin{tabular}{lccc}
\hline & OR & $95 \%$ CI & $p$-value \\
\hline Model 1 & & & \\
Lg10 CCL3 & 1.01 & $0.67-1.53$ & 0.96 \\
Lg10 CCL5 & 1.23 & $0.99-1.53$ & 0.06 \\
Lg10 CCL18 & 1.03 & $0.55-1.93$ & 0.92 \\
Model 2 & & & \\
Lg10 CCL3 & 0.96 & $0.62-1.47$ & 0.84 \\
Lg10 CCL5 & 1.27 & $1.02-1.59$ & $\mathbf{0 . 0 4}$ \\
Lg10 CCL18 & 1.06 & $0.56-2.03$ & 0.86 \\
\hline
\end{tabular}

Model 1: corrected for age and gender; Model 2: corrected for age, gender, diabetes mellitus, BMI, smoking, family history, systolic blood pressure and total cholesterol. $C A D$ coronary artery disease, $C C L C C$ chemokine ligand, $O R$ odds ratio, $C I$ confidence interval $p$-values were two-sided, and a $p$-value below 0.05 was considered statistically significant.

\section{Results}

\section{Study population}

The mean age of the study population was $56 \pm 11$ years, $398(56 \%)$ were males. The indication for CCTA was typical chest pain in $88(12 \%)$, atypical in $310(44 \%)$ and non-anginal complaints in $314(44 \%)$. The mean radiation dose was $5.6 \pm 4.5 \mathrm{mSv}$. The median concentration of CCL3 was $2.7(0.0-7.0) \mathrm{pg} / \mathrm{ml}$, for CCL5 it was 
Table 4 Difference between patients developing an event and patients who do not develop an event

\begin{tabular}{|c|c|c|c|}
\hline & $\begin{array}{l}\text { Patients without event } \\
n=661\end{array}$ & $\begin{array}{l}\text { Patients with event } \\
n=51\end{array}$ & $\begin{array}{l}p \text {-value } \\
-\end{array}$ \\
\hline Age (years) & $56 \pm 10$ & $58 \pm 11$ & 0.29 \\
\hline Male gender & $361(54.6 \%)$ & $37(72.5 \%)$ & 0.01 \\
\hline Smoking & $147(22.2 \%)$ & $22(43.1 \%)$ & 0.002 \\
\hline Diabetes mellitus & $52(7.9 \%)$ & $6(11.8 \%)$ & 0.29 \\
\hline Family history & $252(38.1 \%)$ & $20(39.2 \%)$ & 0.88 \\
\hline Systolic blood pressure $(\mathrm{mm} \mathrm{Hg})$ & $142 \pm 19$ & $143 \pm 20$ & 0.67 \\
\hline $\operatorname{BMI}\left(\mathrm{kg} / \mathrm{m}^{2}\right)$ & $27 \pm 4$ & $27 \pm 4$ & 0.81 \\
\hline Total cholesterol $(\mathrm{mmol} / \mathrm{l})$ & $5.4 \pm 1.1$ & $5.3 \pm 1.3$ & 0.61 \\
\hline HDL cholesterol (mmol/l) & $1.3 \pm 0.6$ & $1.2 \pm 0.4$ & 0.10 \\
\hline LDL cholesterol (mmol/l) & $3.3 \pm 1.0$ & $3.3 \pm 1.2$ & 0.79 \\
\hline Statin use & $244(37.1 \%)$ & $23(45.1 \%)$ & 0.29 \\
\hline Typical chest pain & $81(12.3 \%)$ & $7(13.7 \%)$ & 0.67 \\
\hline Calcium score & $4.6(0.0-103.1)$ & $181.0(20.6-458.2)$ & $<0.001$ \\
\hline Involvement score & $1.0(0.0-3.0)$ & $5.0(2.0-7.0)$ & $<0.001$ \\
\hline CCL3 (pg/ml) & $2.7(0.0-7.0)$ & $2.7(0.0-6.7)$ & 0.96 \\
\hline $\operatorname{CCL} 5\left(\mathrm{pg} / \mathrm{ml} * 10^{2}\right)$ & $34.4(15.0-79.6)$ & $55.6(31.2-115.6)$ & 0.005 \\
\hline $\operatorname{CCL} 18\left(\mathrm{pg} / \mathrm{ml} * 10^{2}\right)$ & $769.0(455.7-1266.0)$ & $941.4(529.9-1272.1)$ & 0.23 \\
\hline hsCRP & $1.4(0.7-3.1)$ & $1.8(1.1-4.1)$ & 0.07 \\
\hline$\geq 50 \%$ stenosis & $162(24.5 \%)$ & $41(80.4 \%)$ & $<0.001$ \\
\hline
\end{tabular}

Values are presented as numbers (\%), means \pm standard deviation or medians (IQR). Significant $p$-values are in bold. $B M I$ body mass index, $C C L$ $\mathrm{CC}$ chemokine ligand, $h \mathrm{C} C R P$ high-sensitive $\mathrm{C}$-reactive protein

Table 5 Multivariable logistic regression analysis to predict for event

\begin{tabular}{|c|c|c|c|}
\hline & OR & $95 \% \mathrm{CI}$ & $p$-value \\
\hline \multicolumn{4}{|l|}{ Model 1} \\
\hline Lg10 CCL3 & 0.81 & $0.41-1.60$ & 0.54 \\
\hline Lg10 CCL5 & 1.71 & $1.07-2.73$ & $\mathbf{0 . 0 3}$ \\
\hline Lg10 CCL18 & 1.33 & $0.45-3.98$ & 0.61 \\
\hline \multicolumn{4}{|l|}{ Model 2} \\
\hline Lg10 CCL3 & 0.88 & $0.43-1.78$ & 0.71 \\
\hline Lg10 CCL5 & 1.60 & $1.01-2.52$ & 0.046 \\
\hline Lg10 CCL18 & 1.35 & $0.45-4.10$ & 0.59 \\
\hline \multicolumn{4}{|c|}{ Model $3(C T \geq 50 \%)$} \\
\hline Lg10 CCL3 & 0.96 & $0.44-2.07$ & 0.91 \\
\hline Lg10 CCL5 & 1.35 & $0.88-2.06$ & 0.17 \\
\hline Lg10 CCL18 & 1.46 & $0.45-4.72$ & 0.53 \\
\hline \multicolumn{4}{|c|}{ Model 4 (involvement score) } \\
\hline Lg10 CCL3 & 0.75 & $0.35-1.59$ & 0.45 \\
\hline Lg10 CCL5 & 1.62 & $1.03-2.57$ & 0.04 \\
\hline Lg10 CCL18 & 1.39 & $0.45-4.34$ & 0.57 \\
\hline
\end{tabular}

Model 1: corrected for age and gender; Model 2: corrected for age, gender, diabetes, BMI, smoking, family history, systolic blood pressure and total cholesterol; Model 3: corrected for age, gender, diabetes, BMI, smoking, family history, systolic blood pressure, total cholesterol and $\geq 50 \%$ stenosis on CCTA; Model 4: corrected for age, gender, diabetes, BMI, smoking, family history, systolic blood pressure, total cholesterol and CCTA involvement score. $C A D$ coronary artery disease, $C C L \mathrm{CC}$ chemokine ligand, $O R$ odds ratio, $C I$ confidence interval 
3614 (1547-8360) $\mathrm{pg} / \mathrm{ml}$ and for CCL18 it was 78,553 $(45,906-126,716) \mathrm{pg} / \mathrm{ml}$ (Fig. 1). Baseline characteristics are further described in Table 1.

\section{Association CCL with CAD}

The serum concentration of CCL5 was significantly higher in patients with $\geq 50 \%$ coronary stenosis, as compared with patients with $<50 \%$ stenosis (Table 1). CCL18 was associated with the calcium score and the segment involvement score (Table 2). As is shown in Table 3, only CCL5 provided independent information to predict a $\geq 50 \%$ coronary stenosis when corrected for traditional risk factors.

\section{CCL concentrations and events}

During a mean follow-up of 26 months, a total of 51 events occurred (3 all-cause mortality, 13 ACS, 10 CABG and 25 PCI). Patients who developed an event during followup were more often males, smokers and had higher calcium and segment involvement scores. Also, their median CCL5 concentration was higher (Table 4). When corrected for traditional risk factors in multivariable regression analysis, CCL5 concentration showed independent predictive value for the occurrence of an event during follow-up (Table 5, model 2). When also corrected for the extent of CAD (adding the segment involvement score, model 4), the independent prognostic value of CCL5 was still present. However, when correcting for the presence of significant stenosis ( $\geq 50 \%$ stenosis, model 3 ), the significant prognostic value of CCL5 disappeared.

\section{Discussion}

In the present study, we investigated the association of CCL3, 5 and 18 with the severity and extent of CAD, as well as their predictive value for the occurrence of primary cardiac events. The main finding was that CCL5 demonstrated independent value to predict the presence of significant CAD. CCL18 was significantly associated with calcium and involvement scores. In addition, CCL5 but not CCL3 and CCL18 provided prognostic value for the occurrence of an event during follow-up, independent of traditional clinical risk factors as well as extent of CAD.

Previously, CCL3, 5 and 18 serum levels have been linked to cardiovascular disease (secondary events in early follow-up, refractory UAP, and ACS) and have been shown to be expressed by atherosclerotic plaque. Also, CCL5 has been related to carotid plaque characteristics [18]. However, evidence on the association of CCL3, 5 and 18 with CAD as well as on the prognostic value for primary ACS is conflicting and limited. Previous studies on CCL3, 5 and
18 have focused on the post-ACS stage, pointing to different roles of CCLs with regard to cardiovascular disease. For instance, CCL5 and CCL18 have shown to be transiently elevated during ischaemia. Especially CCL5 has been strongly associated with platelet activation, and serum levels are altered only transiently in the acute phase of UAP [6]. In our study, CCL5 was independently associated with the presence of $\geq 50 \%$ coronary stenosis, a well-known risk factor for the occurrence of a cardiac event [19, 20]. This is in contrast to a recent, smaller, study investigating the association of CCL5 to $\geq 50 \%$ coronary stenosis based on conventional angiography [21]. In addition, CCL5 serum levels also correlated with the occurrence of cardiac events. This association was independent of traditional risk factors. This independent association remained even when including the extent of CAD, a known risk factor. However, when $\geq 50 \%$ coronary stenosis was added to the model, the significant association disappeared. Possibly, CCL5 is elevated in case of coronary obstruction, and not with extensive CAD only. Conceivably, CCL5 serum levels reflect platelet activation status [6] which could become manifest in obstructive CAD only. Therefore, CCL5 might be promising as an additional risk factor candidate before cardiac imaging diagnostics are applied. However, the value of CCL5 as ACS prognosticator in this study seems limited. Hypothetically, CCL5 might have potential as a therapeutic target as pharmacological inhibition of CCL5 could help to reduce plaque formation. Moreover, previous research showed that CC chemokine receptor 5 deficiency protected men from early myocardial infarction [22], and mice from early plaque formation [23]. On the other hand, previous studies showed a lack of association of CCL5 with future cardiovascular events [10, 11], another even showing a negative association [24]. Regarding these contrasting findings, at present the clinical implications of our findings might be small. We cannot conclude on causality, since we only have described associations. It is possible that CCLs might be elevated in case of an inflammatory state without a causative relationship with coronary atherosclerosis. For example, also hsCRP was associated with CAD.

CCL18 showed a particular association with both the coronary calcium score and the segment involvement score. This might be explained by the fact that CCL18 can directly stimulate fibrosis $[4,25]$, which might have an effect on calcification and more extensive plaque formation. Although CCL18 was not associated with events, it might be associated to the underlying mechanisms such as atherosclerosis progression. CCL18 showed a clear univariable association with extent of CAD as measured by coronary calcium score and involvement score. However, in multivariable analysis, CCL18 was neither independently associated with CAD (not for calcium, segment involvement score or obstructive CAD), nor with the occurrence of events. This 
is in contrast to the results of other prospective studies, in which CCL18 appeared to be an independent predictor of secondary cardiovascular events in the immediate aftermath of AMI or UAP $[8,26]$. In a stable chest pain population, however, CCL18 seems to be dependent on calcification and plaque mass, but not predictive for future events.

The fact that we did not find an association between CCL3 and CAD and/or events, might seem contradictive to previous publications. For instance, higher CCL3 (and CCL5) concentrations were identified in patients with acute myocardial infarction as compared with controls [7]. Also, CCL3 (as well as CCL5 and 18) was independently associated with short-term mortality during follow-up in ACS patients [8]. Importantly, we measured CCL3, 5 and 18 concentrations prior to events. Therefore, collectively the data on CCL3 seem to plead for a role for CCL3 in the acute phase of an ischaemic event, not in the aetiology of ACS. CCL3 seems predominantly ischaemia derived [7] and may be involved in post-ischaemia inflammation, possibly through neutrophil-induced recruitment of monocytes [27].

\section{Limitations}

We studied a relatively large population of patients with chest discomfort, who underwent CCTA to rule out obstructive CAD. Although this population meets our objectives, with the current cohort size, the low event rate of this lowrisk population might have limited the value of longitudinal analyses. The need for revascularisation may be biased by the baseline CCTA. Finally, blood was drawn only at baseline and not during follow-up, which precludes assessment of the dynamics of chemokine patterns prior to an event.

\section{Conclusions}

In patients with stable chest pain, CCL5 and 18 were associated with coronary obstruction and extent of CAD including calcification, respectively. These data suggest CCL5 and 18 are relevant for CAD development. Furthermore, CCL5 appeared to be associated with the occurrence of future cardiac events. This implicates that CCL5 could be a useful marker to predict for CAD and its consequences.

Conflict of interest M.O. Versteylen, M. Manca, I.A. Joosen, D.E. Schmidt, M. Das, L. Hofstra, H.J. Crijns, E.A. Biessen and B.L. Kietselaer state they have no competing interest.

Open Access This article is distributed under the terms of the Creative Commons Attribution 4.0 International License (http:// creativecommons.org/licenses/by/4.0/), which permits unrestricted use, distribution, and reproduction in any medium, provided you give appropriate credit to the original author(s) and the source, provide a link to the Creative Commons license, and indicate if changes were made.

\section{References}

1. Hansson GK, Hermansson A. The immune system in atherosclerosis. Nat Immunol. 2011;12:204-12.

2. Charo IF, Taubman MB. Chemokines in the pathogenesis of vascular disease. Circ Res. 2004;95:858-66.

3. Weber C. Platelets and chemokines in atherosclerosis: partners in crime. Circ Res. 2005;96:612-6.

4. Weber C, Schober A, Zernecke Chemokines A. Key regulators of mononuclear cell recruitment in atherosclerotic vascular disease. Arterioscler Thromb Vasc Biol. 2004;24:1997-2008.

5. Reape TJ, Rayner K, Manning CD, et al. Expression and cellular localization of the CC chemokines PARC and ELC in human atherosclerotic plaques. Am J Pathol. 1999;154:365-74.

6. Kraaijeveld AO, de Jager SC, de Jager WJ, et al. CC chemokine ligand-5 (CCL5/RANTES) and CC chemokine ligand-18 (CCL18/ PARC) are specific markers of refractory unstable angina pectoris and are transiently raised during severe ischemic symptoms. Circulation. 2007;116:1931-41.

7. de Jager SC, Kraaijeveld AO, Grauss RW, et al. CCL3 (MIP-1 alpha) levels are elevated during acute coronary syndromes and show strong prognostic power for future ischemic events. J Mol Cell Cardiol. 2008;45:446-52.

8. de Jager SC, Bongaerts BW, Weber M, et al. Chemokines CCL3/MIP1alpha, CCL5/RANTES and CCL18/PARC are independent risk predictors of short-term mortality in patients with acute coronary syndromes. PLoS ONE. 2012;7:e45804.

9. Hagg DA, Olson FJ, Kjelldahl J, et al. Expression of chemokine (C-C motif) ligand 18 in human macrophages and atherosclerotic plaques. Atherosclerosis. 2009;204:e15-20.

10. Correia LC, Andrade BB, Borges VM, et al. Prognostic value of cytokines and chemokines in addition to the GRACE Score in non-ST-elevation acute coronary syndromes. Clin Chim Acta. 2010;411:540-5.

11. Herder C, Peeters W, Illig T, et al. RANTES/CCL5 and risk for coronary events: results from the MONICA/KORA Augsburg casecohort, Athero-Express and CARDIoGRAM studies. PLoS ONE. 2011;6:e25734.

12. Canoui-Poitrine F, Luc G, Mallat Z, et al. Systemic chemokine levels, coronary heart disease, and ischemic stroke events: the PRIME study. Neurology. 2011;77:1165-73.

13. Shrimpton PC. Assessment of patient dose in CT: appendix C European guidelines for multislice computed tomography. European Commission project MSCT: CT safety \& efficacy - a broad perspective. 2014. http://www.msct.eu/PDF_FILES/Appendix \%20paediatric\%20CT\%20Dosimetry.pdf. Accessed March 2014.

14. Agatston AS, Janowitz WR, Hildner FJ, Zusmer NR, Viamonte M Jr., Detrano R. Quantification of coronary artery calcium using ultrafast computed tomography. J Am Coll Cardiol. 1990;15:827-32.

15. Austen WG, Edwards JE, Frye RL, et al. A reporting system on patients evaluated for coronary artery disease. Report of the Ad Hoc Committee for Grading of Coronary Artery Disease, Council on Cardiovascular Surgery, American Heart Association. Circulation. 1975;51:5-40.

16. Min JK, Shaw LJ, Devereux RB, et al. Prognostic value of multidetector coronary computed tomographic angiography for prediction of all-cause mortality. J Am Coll Cardiol. 2007;50:1161-70.

17. Braunwald E, Antman EM, Beasley JW, et al. ACC/AHA 2002 guideline update for the management of patients with unstable angina and non-ST-segment elevation myocardial infarction summary article: a report of the American College of Cardiology/American Heart Association task force on practice guidelines 
(Committee on the Management of Patients With Unstable Angina). J Am Coll Cardiol. 2002;40:1366-74.

18. Virani SS, Nambi V, Hoogeveen R, et al. Relationship between circulating levels of RANTES (regulated on activation, normal T-cell expressed, and secreted) and carotid plaque characteristics: the Atherosclerosis Risk in Communities (ARIC) Carotid MRI Study. Eur Heart J. 2011;32:459-68.

19. Min JK, Dunning A, Lin FY, et al. Age- and sex-related differences in all-cause mortality risk based on coronary computed tomography angiography findings results from the International Multicenter CONFIRM (Coronary CT Angiography Evaluation for Clinical Outcomes: An International Multicenter Registry) of 23,854 patients without known coronary artery disease. J Am Coll Cardiol. 2011;58:849-60.

20. Versteylen MO, Joosen IA, Winkens MH, et al. Combined use of exercise electrocardiography, coronary calcium score and cardiac CT angiography for the prediction of major cardiovascular events in patients presenting with stable chest pain. Int J Cardiol. 2012;167:121-5.

21. Niki T, Soeki T, Yamaguchi K, et al. Elevated concentration of interferon-inducible protein of $10 \mathrm{kD}$ (IP-10) is associated with coronary atherosclerosis. Int Heart J. 2015;56:269-72.
22. Gonzalez P, Alvarez R, Batalla A, et al. Genetic variation at the chemokine receptors CCR5/CCR2 in myocardial infarction. Genes Immun. 2001;2:191-5.

23. Potteaux S, Combadiere C, Esposito B, et al. Role of bone marrowderived CC-chemokine receptor 5 in the development of atherosclerosis of low-density lipoprotein receptor knockout mice. Arterioscler Thromb Vasc Biol. 2006;26:1858-63.

24. Cavusoglu E, Eng C, Chopra V, Clark LT, Pinsky DJ, Marmur JD. Low plasma RANTES levels are an independent predictor of cardiac mortality in patients referred for coronary angiography. Arterioscler Thromb Vasc Biol. 2007;27:929-35.

25. Atamas SP, Luzina IG, Choi J, et al. Pulmonary and activationregulated chemokine stimulates collagen production in lung fibroblasts. Am J Respir Cell Mol Biol. 2003;29:743-9.

26. De Sutter J, Struyf S, Van de Veire NR, Philippe J, De Buyzere M, Van Damme J. Cardiovascular determinants and prognostic significance of CC chemokine ligand-18 (CCL18/PARC) in patients with stable coronary artery disease. J Mol Cell Cardiol. 2010;49:894-6.

27. Soehnlein O, Lindbom L, Weber C. Mechanisms underlying neutrophil-mediated monocyte recruitment. Blood. 2009;114:4613-23. 